\title{
Local Compression in Automated Breast Ultrasound in the Mammographic Geometry
}

\author{
Paul L. Carson ${ }^{1,2}$, Boyun Wang ${ }^{3,4}$, Gerald L. LeCarpentier ${ }^{1}$, Mitchell M. Goodsitt ${ }^{1}$, Chris Lashbrook ${ }^{1}$, \\ Renee Pinsky ${ }^{1}$, Ganesh Narayanasamy ${ }^{1,5,6}$, J. Brian Fowlkes ${ }^{1,2}$, Kazuhiro Saitou ${ }^{3}$ \\ ${ }^{1}$ Department of Radiology, University of Michigan, Ann Arbor, MI, USA \\ ${ }^{2}$ Department of Biomedical Engineering, University of Michigan, Ann Arbor, MI, USA \\ ${ }^{3}$ Department of Mechanical Engineering, University of Michigan, Ann Arbor, MI, USA \\ ${ }^{4}$ Department Electrical Engineering, University of Michigan, Ann Arbor, MI, USA \\ ${ }^{5}$ Department of Applied Physics, University of Michigan, Ann Arbor, MI, USA \\ ${ }^{6}$ Radiation Medicine, University of Kentucky Medical Center, Lexington, KY, USA
}

\begin{abstract}
Background, Motivation and Objective: Automated ultrasound scanning (AUS) of the breast has developed more slowly than anticipated. The main limitation, beyond achieving adequate acoustic coupling to the breast, has been excessive shadow artifacts, as reflecting structures at acute angles to the ultrasound beam are not flattened by the transducer as well as in manual scanning. We believe that imaging of the breast in near mammographic compression provides much of the needed flattening. The question under initial study in this effort is, whether in breast AUS under very light mammographic compression, local compression by the transducer might flatten the acutely oriented structures further and reduce the acoustic path length to key structures in the breast. We suspect these improvements will be possible without distorting the breast so dramatically that the lesion registration advantages of scanning the breast in the same system as mammography or digital breast tomosynthesis (DBT) are not realized. Preliminary tests are reported here, as well as design of a system for a more refined human study. Statement of Contribution/Methods: Initial imaging tests were performed in our combined AUS/DBT system. A fiber mesh, loosened slightly in its frame, replaced the standard plastic mammography compression paddle. The transducer, in contact with the mesh and the breast, was translated by motors. The compression force of the linear array transducer on its vertical was manually controlled. Breast phantoms and the breasts of three women were scanned with usual compression by the mesh paddle and then with less global, but added local, compression. Results: Examples of flattened structures were observed more brightly in the locally compressed breasts, and acoustic paths longer than $35 \mathrm{~mm}$ were reduced, by $\sim 10 \mathrm{~mm}$. In many areas image penetration was $3 \mathrm{~cm}$ greater. In one case, image volumes w/wo local compression were spatially aligned by nonlinear image registration software. Discussion and Conclusions: Visual indicators of image features expected to provide improved ultrasonic imaging were observed with local compression and lateral movement of tissues appeared acceptable. These results motivated design and construction of an apparatus to make local compression practical and safe. It utilizes joystick control of the vertical compression force during scanning, realized by pneumatic actuators attached to the transducer. The air pressure applied to these actuators is also applied to actuators in the joystick for
\end{abstract}

force feedback to the operator. Two miniature vibrators attached to the joystick provide vibrotactile feedback of the reaction torques computed from the measurements of 6 force sensors on the transducer holder. The fail-safe system design insures no pneumatic compression force application to the breast in case of power loss or emergency shutdown.

Keywords- breast imaging, local compression, remote control, automated scanning

\section{INTRODUCTION}

Fully automated ultrasound scanning (AUS) of the breast, while currently an important and promising topic, has been less successful than desired. The main limitation, after achieving adequate acoustic coupling to and coverage of the breast, has been excessive shadow artifacts. Reflecting structures at acute angles to the ultrasound beam cause this shadowing and reduced penetration. We believe that imaging of the breast in full mammographic compression, or in slightly less than full mammographic compression, provides much of the needed flattening of these structures compared with the water path scanning of ultrasonic CT $[1,2]$. Still, the flattening effect by the transducer or compression plate is probably not as great as in manual scanning [3.4] or automated scanning with manual control of contact pressure [5]. The questions under initial study in this effort is, whether in automated scanning of the breast under light mammographic compression, local compression by the transducer might flatten the acutely oriented structures further and reduce the acoustic path length to key structures in the breast. We suspect these improvements will be possible without distorting the breast so dramatically that one cannot realize the dual-modality lesion registration advantages of scanning the breast with ultrasound in the same geometry, as mammography or 3D x-ray digital breast tomosynthesis (DBT). Preliminary tests of these concepts 
are reported here, as well as the design of a system for a more complete human study.

\section{MATERIALS AND MethodS}

\section{A. Preliminary evaluation with manual contact pressure for local compression}

The current research combined AUS/DBT system was developed by GE Global Research, Niskayuna, NY, in collaboration with scientists at the University of Michigan Radiology, Ann Arbor. It utilizes a Logiq 9 US system with an M12 array by GE Health Systems, Milwaukee, WI. This system functions more effectively with a mesh paddle (Figure 1) than with the solid paddle employed over the past few years [6,7]. When loosened, the mesh allows substantial local compression by the motor-scanned transducer. A phantom and three asymptomatic volunteers were scanned in the combined system without and with various levels of local compression. In these minimally aberrating breasts, a shorter distance of breast structures to the transducer served as an indicator of potential improvements in image quality.

\section{B. New system for operator assisted local compression}

The new system has been designed to implement localized compression under operator control during the motorized $x-y$ scanning, realized by pneumatic actuators attached to the transducer as illustrated in Figure 2. The small volume of gas allows for a safe, pneumatic actuation. Springs countering the pistons and other fail-safe design will ensure no pneumatic compression force application to the breast in case of power loss or emergency shutdown.

\section{RESUltS AND DISCUSSION}

\section{A. Effects of manual local compression}

Figures $3 \mathrm{~A}$ and $3 \mathrm{~B}$ compare approximately equivalent image planes from AUS scans with very modest spring loaded local compression and with fairly strong manual compression, respectively. Nonlinear, 3D image registration was applied to volume A to register with volume B. An example of the results is shown in Figure 3C. This success in evident in comparing Figure 3C with Figures $3 \mathrm{~A}$ and $3 \mathrm{~B}$. The distance scale in $\mathrm{cm}$ for all three images is on the right, with transmit focal lines marked with arrows. One can see that the more substantial local compression reduced the acoustic path length to deeper structures by approximately $6 \mathrm{~mm}$.

In other locations in this and the other two image series, a few flattened structures were observed more brightly in the locally compressed breasts. Acoustic path lengths to breast structures lying greater than $35 \mathrm{~mm}$ deep were reduced, often by $\sim 10 \mathrm{~mm}$ particularly when compared with no local compression. In many areas image penetration allowed visualization of structures up to $3 \mathrm{~cm}$ deeper. The mesh paddle frame was not moved between AUS sweeps in a series of compressions. Lateral movement of tissues between local and global mesh-compressed breasts appeared acceptably smooth and small, such as to probably allow reasonable spatial correlation of breast tissues in the AUS and DBT

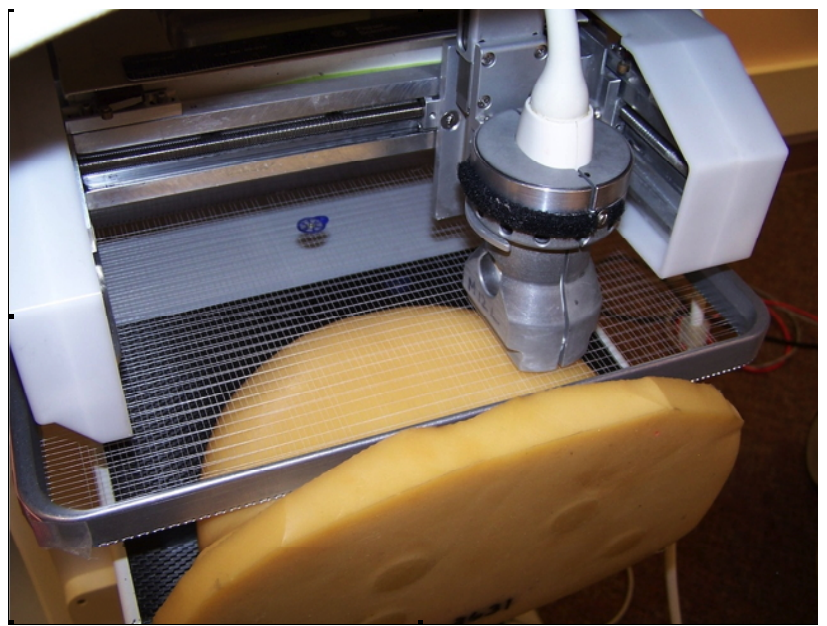

Figure 1. Existing Ultrasound system for scanning a breast phantom, stabilized by a fiber mesh and its frame over the x-ray detector (black). After x-ray TM, the US scanning from is rotated into place over the breast. Coupling gel is not yet applied. The transducer's vertical position can be fixed, spring loaded, or manually controlled for local compression.

image volumes acquired in the same initial compression. For more precise co-registration of AUS and DBT image volumes, scans can be acquired without and with local compression, and image based registration performed between two AUS volumes in order to register the locally compressed AUS to the DBT.

\section{B. System for operator assisted local compression}

The local compression system utilizes joystick control of the vertical compression force during scanning, realized by pneumatic actuators attached to the transducer. The same air pressure applied to these actuators is applied to actuators in the joystick for force feedback to the operator. Two miniature vibrators attached to the joystick provide vibrotactile feedback of the reaction forces and torques are computed from the measurements of 6 force sensors on the transducer holder. A $\mathrm{CAD}$ drawing of the transducer mount with pneumatic pistons to assist the operator in local compression is shown in Figure 4. Additional refinement of this prototype is required before use in human studies.

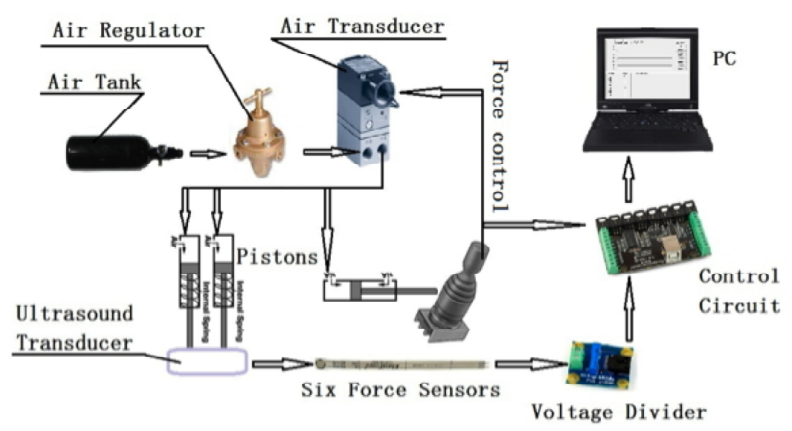

Figure 2. Schematic of local compression system design. 


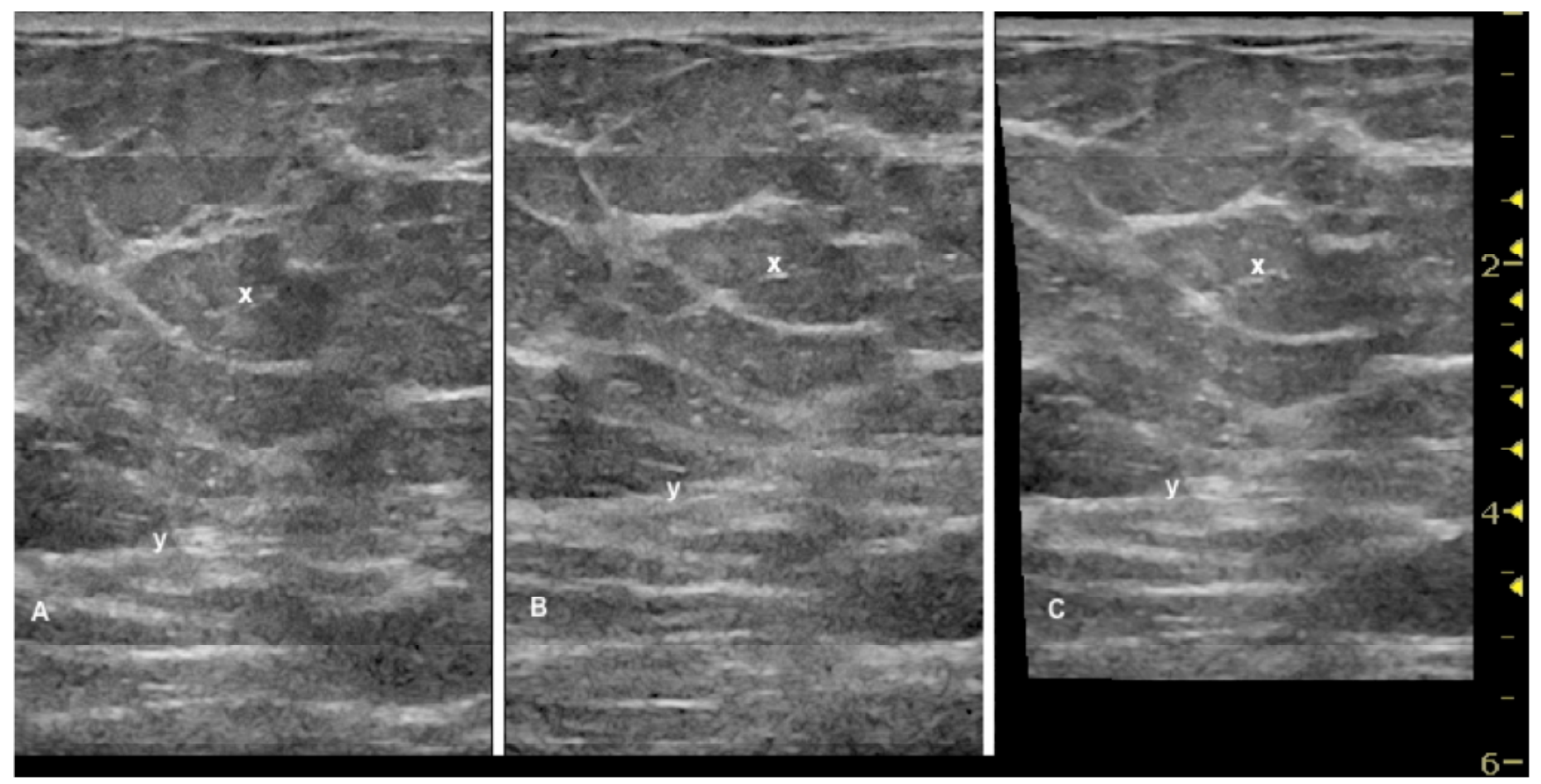

Figure 3. AUS scans of normal volunteer with mesh paddle compression: (A) With slight additional compression by spring loaded transducer; (B) With substantial manual compression by transducer; (C) Is (A) after image based registration to (B). Approximately equivalent points are marked with $\mathrm{x}$ and $\mathrm{y}$.

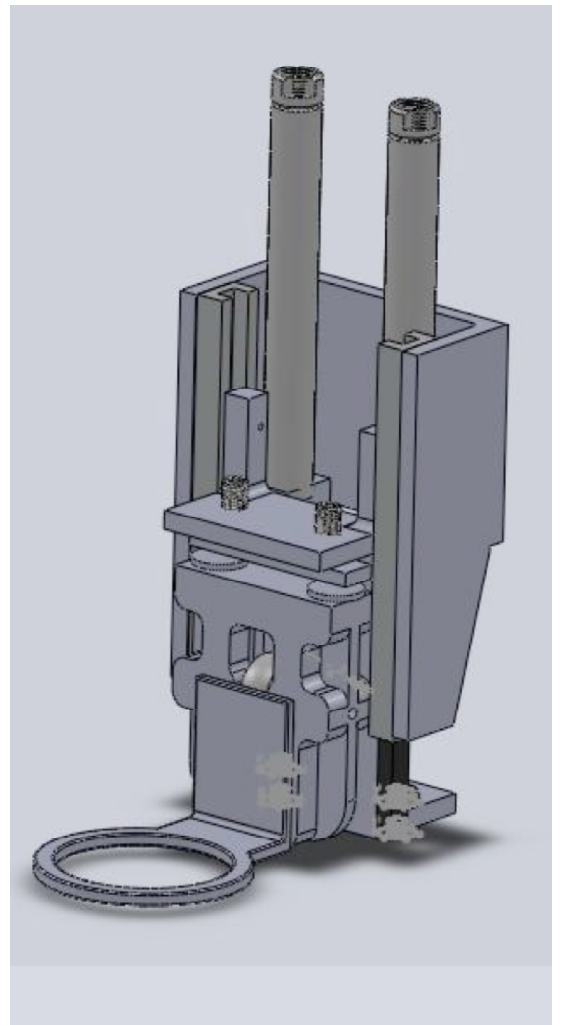

Figure 4. New transducer holder with pneumatic position control.

\section{CONCLUSION}

In limited preliminary phantom and patient studies, visual indicators of improved ultrasound image quality were observed with local compression. An apparatus capable of making more extensive tests of local compression both practical and safe has been designed and an initial implementation constructed. It is anticipated that the increase in ultrasound image quality of deeper structures with localized compression will justify the hopefully modest displacement of the tissues from their locations at the time of $x$-ray image acquisition and not impair co-registration of lesions with the combined AUS/DBT system.

\section{ACKNOWLEDGMENT}

This work was supported in part by NIH Grants 2R01CA91713 and 3R01CA91713S.

\section{REFERENCES}

[1] K.S. Callahan, D.T. Borup, S.A. Johnson, J. Wiskin, Y. Parisky, "Transmission breast ultrasound imaging: Representative case studies of speed of sound and attenuation of sound computed tomographic images," Am. J. Clin. Oncol. 30, 458-459 (2007).

[2] N. Duric, P. Littrup, L. Poulo, A. Babkin, R. Pevzner, E. Holsapple, O. Rama, C. Glide, "Detection of breast cancer with ultrasound tomography: First results with the Computed Ultrasound Risk Evaluation (CURE) prototype," Med. Phys. 34, 773-785 (2007). 
[3] Halliwell $\mathrm{M}$ and Curtis $\mathrm{S}$, "Ultrasound Breast Imaging: A 3dimensional approach, work-in-progress," Toshiba Review: Visions/Ultasound, 67-75, (2008).

[4] J.A. Shipley, F.A. Duck, D.A. Goddard, M.R. Hillman, M. Halliwell, M.G. Jones, B.T. Thomas, "Automated quantitative volumetric breast ultrasound data-acquisition system," Ultrasound Med. Biol. 31, 905917 (2005).

[5] Kelly KM, Dean J, Comulada WS, Lee SJ, " Breast cancer detection using automated whole breast ultrasound and mammography in radiographically dense breasts," Eur Radiol 20, 734-742, (2010).

[6] Sinha S.P., M.M. Goodsitt, M.A. Roubidoux, R.C. Booi, G.L. LeCarpentier GL, C.R. Lashbrook, et al., "Automated Ultrasound Scanning on a Dual Modality Breast Imaging System", Journal of Ultrasound in Medicine 26: 645-655, (2007).
[7] Sinha S.P .,M.A. Roubidoux, M.A. Helvie, A.V. Nees, M.M. Goodsitt $\mathrm{MM}$, et al., "Multi-modality 3D breast imaging with X-Ray tomosynthesis and automated ultrasound", Conf Proc IEEE Eng Med Biol Soc.:1335-8, (2007).

[8] Narayanasamy G, LeCarpentier GL, Roubidoux M, Fowlkes JB, Schott $\mathrm{AF}$, Carson PL, "Spatial registration of temporally separated whole breast 3D ultrasound images," Med. Phys. 36, 4288-4300, PMC2749445, (2009).C. Coussios and R. Roy, "Applications of Acoustics and Cavitation to Noninvasive Therapy and Drug Delivery," Annu. Rev. Fluid Mech. Vol. 40, pp. 395-420, 2008. 